\title{
COMPLETENESS OF IMAGING SURVEYS FOR ECCENTRIC EXOPLANETS
}

\author{
STEPHEN R. KANE \\ NASA Exoplanet Science Institute, Caltech, MS 100-22, 770 South Wilson Avenue, Pasadena, CA 91125, USA; skane@ipac.caltech.edu \\ Received 2012 November 28; accepted 2013 January 31; published 2013 March 1
}

\begin{abstract}
The detection of exoplanets through direct imaging has produced numerous new positive identifications in recent years. The technique is biased toward planets at wide separations due to the difficulty in removing the stellar signature at small angular separations. Planets in eccentric orbits will thus move in and out of the detectable region around a star as a function of time. Here we use the known diversity of orbital eccentricities to determine the range of orbits that may lie beneath the detection threshold of current surveys. We quantify the percentage of the orbit that yields a detectable signature as a function of semimajor axis, eccentricity, and orbital inclination and estimate the fraction of planets which likely remain hidden by the flux of the host star.
\end{abstract}

Key words: planetary systems - techniques: high angular resolution - techniques: radial velocities

\section{INTRODUCTION}

The diversity of exoplanetary systems has been revealed through a variety of detection techniques which are sensitive to different kinds of planets. The majority of planets have been detected indirectly via the radial velocity (RV) and transit methods, but the technique of direct imaging has made important contributions by detecting young planets at wide separations ( $\gtrsim 10 \mathrm{AU})$. Direct imaging is a difficult technique to execute and requires the use of adaptive optics and high-contrast imaging to distinguish the flux of the planet from that of the host star (Oppenheimer \& Hinkley 2009). The characteristic properties of atmospherically induced speckle noise can be treated using adaptive optics techniques (Racine et al. 1999; Macintosh et al. 2007). Speckle noise due to telescope/optics imperfections require more advanced techniques such as angular differential imaging (ADI; Marois et al. 2005, 2006; Lafrenière et al. 2007c), spectral differential imaging (SDI; Lafrenière et al. 2007b; Vigan et al. 2010), and polarimetric differential imaging (PDI; Quanz et al. 2012). The list of both technical and scientific achievements from exoplanet imaging surveys is far too exhaustive to describe here in detail, but include such milestone exoplanet discoveries as beta Pic b (Lagrange et al. 2010; Currie et al. 2011), the HR 8799 planetary system (Marois et al. 2008, 2010; Hinkley et al. 2011; Currie et al. 2012b), and Fomalhaut b (Kalas et al. 2008; Currie et al. 2012a).

The star-planet separations explored by the imaging and $\mathrm{RV}$ techniques are complementary to each other since the RV method is biased toward shorter orbital periods. Although the imaging technique requires considerable follow-up observations to constrain the orbits of the planets detected, $\mathrm{RV}$ planets provide complete Keplerian orbital solutions at the time of discovery which may be used to study the overall statistics of exoplanetary systems. The eccentricity distribution has been investigated for RV planets (Shen \& Turner 2008; Hogg et al. 2010) and also compared to that of the Kepler candidates (Moorhead et al. 2011; Kane et al. 2012). The origin of the observed distribution has also been studied in the context of planet formation scenarios and early dynamical evolution (Namouni 2005; Ribas \& MiraldaEscudé 2007; Ford \& Rasio 2008; Malmberg \& Davies 2009; Kley \& Nelson 2012). If this eccentricity distribution also applies to the kinds of planets detected via direct imaging, then the detectability of those planets will depend on where they are in their orbit since the star-planet separation is time-dependent (Brandeker et al. 2006; Bonavita et al. 2012).

Here we investigate the effects of exoplanet orbital eccentricity on the detection efficiency of imaging surveys. We describe the constraints on observing planets in eccentric orbits depending upon the sensitivity of direct imaging experiments to the inner region of the system and the cadence of the observations. We calculate the percentage of the orbit for which the planet will be detectable as a function of eccentricity and the sensitivity threshold. We study the known eccentricity distribution as a function of semimajor axis and star-planet angular separation. Finally, we use this distribution to estimate the detection efficiency of direct imaging for eccentric exoplanets.

\section{OBSERVATIONAL CONSTRAINTS}

In addition to the science requirements of imaging experiments for achieving high-contrast observations that allow one to detect the extreme star-planet flux ratio, one also needs to contend with the limit of how close to the host star can reasonably be probed for planets. The angular resolution needed for achieving this is limited by the diffraction-limited resolution where the objects are separated by $\lambda / D$ where $D$ is the aperture diameter of the telescope. In practice, there are further techniques that may be brought to bear on the problem that can further suppress the starlight and enhance the contrast ratio. These techniques vary enormouslessly in both their effectiveness and their number. Here we quantify the problem in terms of a uniform inner region around a star for which planet detections are inaccessible. We refer to this region as an exclusion zone with radius $r_{e}$. Although a practical exclusion zone will be a quite complicated non-uniform pattern due to diffraction and speckle effects, this allows us specify a lower-limit that may be adapted to any particular experiment.

The problem of detecting eccentric exoplanets for which the star-planet separation is time-dependent is portrayed in Figure 1. The shaded region represents the exclusion zone for a given star. The three orbits shown represent three Keplerian orbits that have identical components with the exception of eccentricity, shown here for $e=0.3,0.6$, and 0.9. The argument of periastron is set to $\omega=315^{\circ}$ for this example. The semimajor axis in all cases is set to $a=r_{e}$, such that a circular orbit would lie on the threshold of detectability. The orbits 


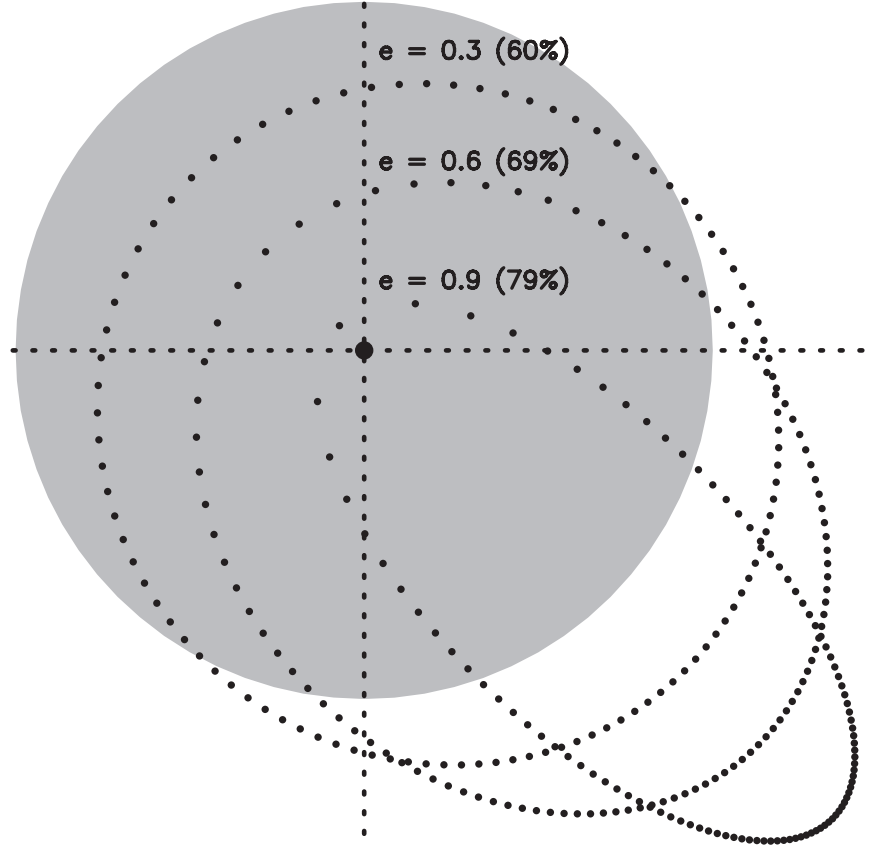

Figure 1. Three example orbits for a planet in orbit around a direct imaging target. The shaded region indicates the angular region around the star for which the example imaging experiment is insensitive to positive planet detections (exclusion zone). The orbits each have a semimajor axis equal to the radius of the exclusion zone and have eccentricities of $0.3,0.6$, and 0.9 . The orbits are shown as points that indicate the planet location in equal time increments. The numbers in parentheses show the percentage of the orbit that lies outside the exclusion zone.

are shown as points that indicate the planet location in equal time increments to illustrate the relative amount of time the planet spends at different phases of its eccentric orbit. In this example, there are clear advantages for highly eccentric planets whose orbits result in a larger angular separation from the host star and a larger percentage of the orbit outside of the exclusion zone.

The percentage of the orbit during which the planet is in the detectable region depends on the eccentricity and semimajor axis relative to $r_{e}$. This is shown in Figure 2 for four different values of $a$ (in units of $r_{e}$ ). For $a<r_{e}$ there are large swathes of eccentricities for which the orbit never exits the exclusion zone. The limit at which the planet never exits the exclusion zone is found from evaluating the star-planet separation at apastron, that is where $a(1+e)<r_{e}$. As $e$ approaches unity, this limit is located at $a=0.5 r_{e}$. A similar bound on a semimajor axis beyond which the planet never enters the exclusion zone is more open-ended since the periastron boundary condition of $a(1-e)>r_{e}$ approaches infinity as $e$ approaches unity.

Another observation constraint is that of the observing cadence. The planet is moving at its slowest speed when the star-planet separation is approaching maximum. Thus even sparse sampling should in principle allow the tracking of the planetary orbit. There are, however, two things to consider here. Firstly, if the movement of the planet is less than the positional accuracy of the planetary point-spread function, then this will create degeneracy in the derived Keplerian solution for the planet. On the other hand, too sparse sampling could result in the planet being missed in future (or past) observations if the apastron is near the detection threshold, such as the $a=0.6 r_{e}$ case shown in Figure 2. Note that the examples shown here aid in definining the problem posed by eccentric orbits, but assume

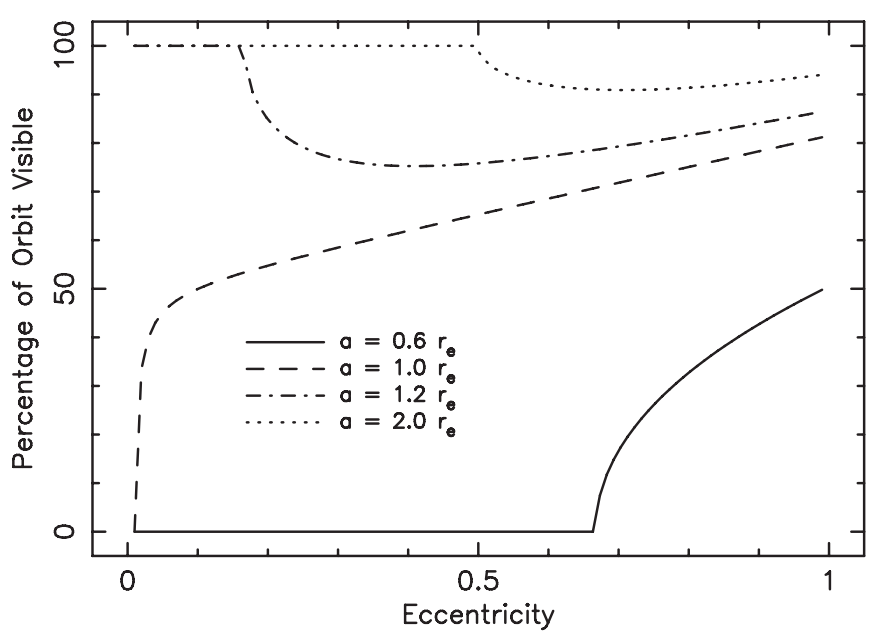

Figure 2. Dependence of the percentage of an orbit spent outside the exclusion zone on the orbital eccentricity for four different semimajor axis. The semimajor axes are in units of the exclusion zone radius, $r_{e}$.

face-on orbits for the specific numbers calculated. We discuss the effects of inclination in Section 4.

\section{ECCENTRICITY DISTRIBUTION AT LONG PERIODS}

Here we briefly discuss the eccentricity distribution of the known exoplanets, particularly at relatively long periods. To do this, we utilize the orbital parameters stored in the Exoplanet Data Explorer $^{1}$ (Wright et al. 2011). We extracted the data of 513 planets, along with the host star properties, with data current as of 2012 November 20. These data include all planets with the necessary Keplerian orbital solutions as well as stellar distances.

In Figure 3 we have plotted these data to show the distributions of eccentricity values. The left plot shows this distribution as a function of semimajor axis, where we have logarithmically scaled the size of the points depending on the planetary mass. The relative dearth of planets between $0.1 \mathrm{AU}$ and 1.0 AU is well-known, noted, for example, by Butler et al. (2006). As is clear from the plot, the mean eccentricity increases with semimajor axis and is $0.06,0.24$, and 0.27 for semimajor axis ranges of $0.0-0.1,0.1-1.0$, and $1.0-2.0$, respectively. What is not so clear is the increase of planet mass with eccentricity; 2.5 , 3.6, and 5.4 Jupiter masses for eccentricity ranges of $0.0-0.3$, $0.3-0.6$, and $0.6-1.0$, respectively. This is of particular relevance to the imaging surveys since they are more sensitive to more massive planetary companions.

In the right panel of Figure 3 we have plotted the eccentricity against the calculated angular projected separation of the planet from the star. This angular separation is calculated for a faceon orbit when the planet is located at maximum separation (apastron). For a Keplerian orbit, the time-dependent star-planet separation, $r$, is given by

$$
r=\frac{a\left(1-e^{2}\right)}{1+e \cos f},
$$

where $f$ is the true anomaly. The general expression (in radians) for the angular separation as a function of time is then

$$
\Delta \theta=\frac{r}{d}\left(\cos ^{2}(\omega+f)+\sin ^{2}(\omega+f) \cos ^{2} i\right)^{\frac{1}{2}},
$$

where $\omega$ is the periastron argument, $i$ is the orbital inclination, and $d$ is the star-observer distance (Kane \& Gelino 2011).

\footnotetext{
1 http://exoplanets.org/
} 

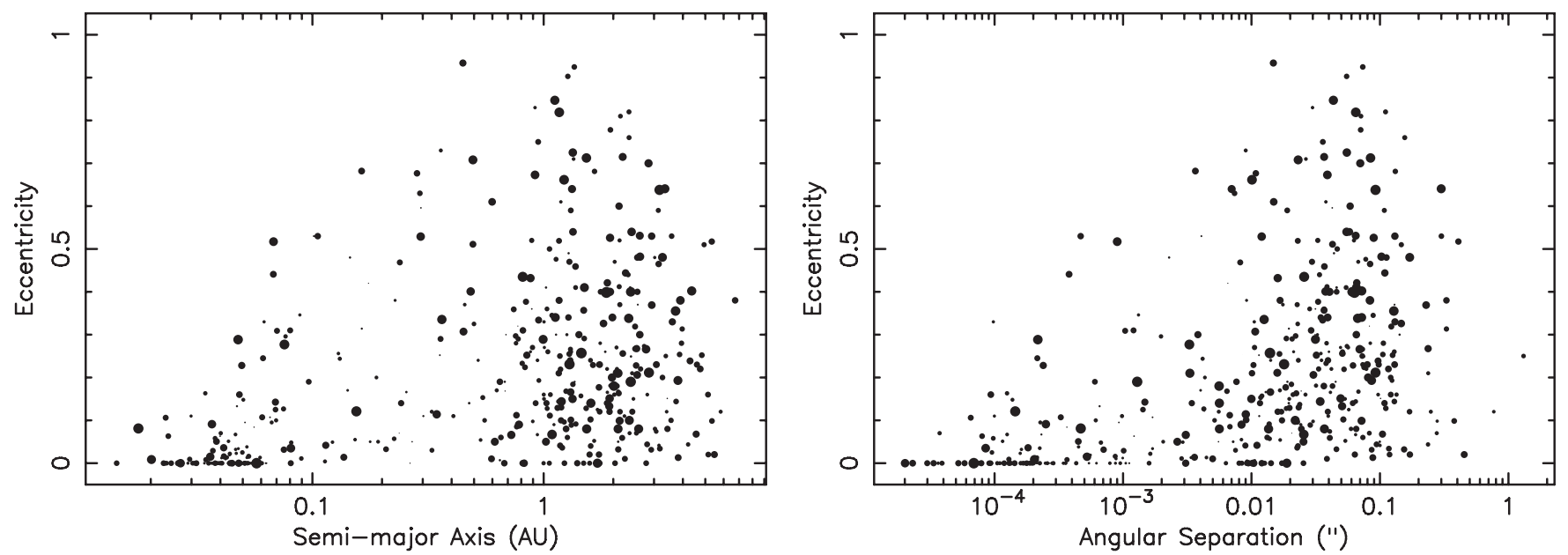

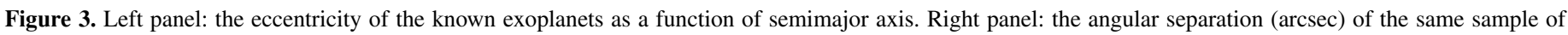
planets at apastron and assuming a face-on orbit $\left(i=0^{\circ}\right)$. In each plot, the size of each plotted point is logarithmically proportional to the mass of the planet.

This angular separation is a useful diagnostic to show which of the known exoplanets may yield positive results from a certain imaging experiment. What is clear is that many of those planets that fall into a detectable range of angular separations will be at the apastron of an eccentric orbit.

\section{DETECTION EFFICIENCY FOR ECCENTRIC ORBITS}

We now utilize the components of the previous sections to calculate the expected fraction of planets that will be detectable from images experiments. To do this, we require the inclusion of the effects of inclination since this can have a large effect on the projected separation between the star and planet. Calculations from Equation (2) are combined with those of a Keplerian orbital solution to compute the projected separation as a function of orbital phase, where phase zero is the location in the orbit where $\omega+f=270^{\circ}$. We also set $\omega=315^{\circ}$ and $a=r_{e}$. The choice $\omega$ is to demonstrate the asymmetry that can result when considering eccentric orbits, a more thorough discussion with application to photometric phase curves of which may be found in Kane \& Gelino (2011). We show the results of these calculations in Figure 4 where we use three different values for eccentricity ( $e=0.3,0.6$, and 0.9$)$ and inclination $\left(i=0^{\circ}\right.$ (faceon), $45^{\circ}$, and $90^{\circ}$ (edge-on)). The shaded region indicates the exclusion zone (as for Figure 1). The eccentricity, inclination, and percentage of the orbit that lies outside the exclusion zone, respectively, are given at the top of each subplot. In general, the most favorable condition for detection is that for which inclinations are close to face-on, although high-eccentricity orbits suffer less in this regard when orbits become close to edgeon. Depending on the asymmetry in the projected separation (sensitively dependent on $\omega$ ), increased values of $a$ will result in multiple opportunities for detection as the orbit moves in and out of the exclusion zone.

To quantify the effect of all these aspects on the detection efficiency of imaging experiments, we performed a detailed Monte Carlo simulation. This simulation calculates the percentage of the orbit which lies outside the exclusion zone for each of the known exoplanets described in Section 3. We calculate this a function of the exclusion zone expressed as an angular separation which uses the known distance to the star. We also consider three inclinations for all planets; $i=0^{\circ}, 45^{\circ}$, and $90^{\circ}$. We then randomly determine if the planet lies outside the exclusion zone, thus using the percentage of time outside the exclusion zone as a probability distribution. The results of this simulation are shown in Figure 5 where we have calculated the percentage of the planets recovered in each of the simulations for each exclusion radius. We consider the range of exclusion radii from 0.2 .01 to $0^{\prime \prime} .2$, a range for which many of the known exoplanets, particularly those at short periods, will remain elusive. For comparison, the angular separation of HR 8799 e from the host star is 0.'37 (Marois et al. 2010), and the inner working angle of selected future ground-based imaging instruments described by Beichman et al. (2010) range from 0.'03 to 0 . 17 . The results of this simulation support the conclusion from Figure 4 that face-on inclinations are more favorable for detection. More importantly though, it demonstrates the dramatic dependence on the exclusion radius for the known exoplanets. This dependence on the exclusion radius is true even if all the orbits are circular. Note that assuming an isotropic distribution of orbital inclinations by randomly selecting the inclination for each planet in the Monte Carlo simulation results in a dependence that is roughly equivalent to the $i=45^{\circ}$ case shown in Figure 5.

Finally, we performed a simulation that uses the observed eccentricity distribution described in Section 3. We first used the eccentricities of the planets between 0.0 and $0.1 \mathrm{AU}$ to represent near circular orbits. This consists of 165 planets which we use as the seed for the Monte Carlo simulation whilst fixing all semimajor axes to a given value. As for the previous simulation, we choose random points in the orbital phase for each planet and determine if the planet is detected (outside the exclusion radius) or undetected (inside the exclusion radius). For semimajor axes of $a=1.0 r_{e}, 76 \%$ of planets are detected, whereas for $a=1.5 r_{e}, 100 \%$ of planets are detected. We then repeated this simulation by using the eccentricities of the planets that lie between 1.0 and $2.0 \mathrm{AU}$; a sample that is more representative of planets at longer orbital periods where imaging experiments are more sensitive. For semimajor axes of $a=1.0 r_{e}, 62 \%$ of these planets are detected, and for $a=1.5 r_{e}, 96 \%$ of planets are detected. This is consistent with the results shown in Figure 2 and indicates that the vast majority of eccentric planets with $a>1.5 r_{e}$ should be detectable at any given time. Note that this assumes that the eccentricity distribution between 1.0 and 2.0 AU continues at longer orbital periods. Figure 3 suggests that the mean eccentricity may decline beyond 2.0 AU. However, 

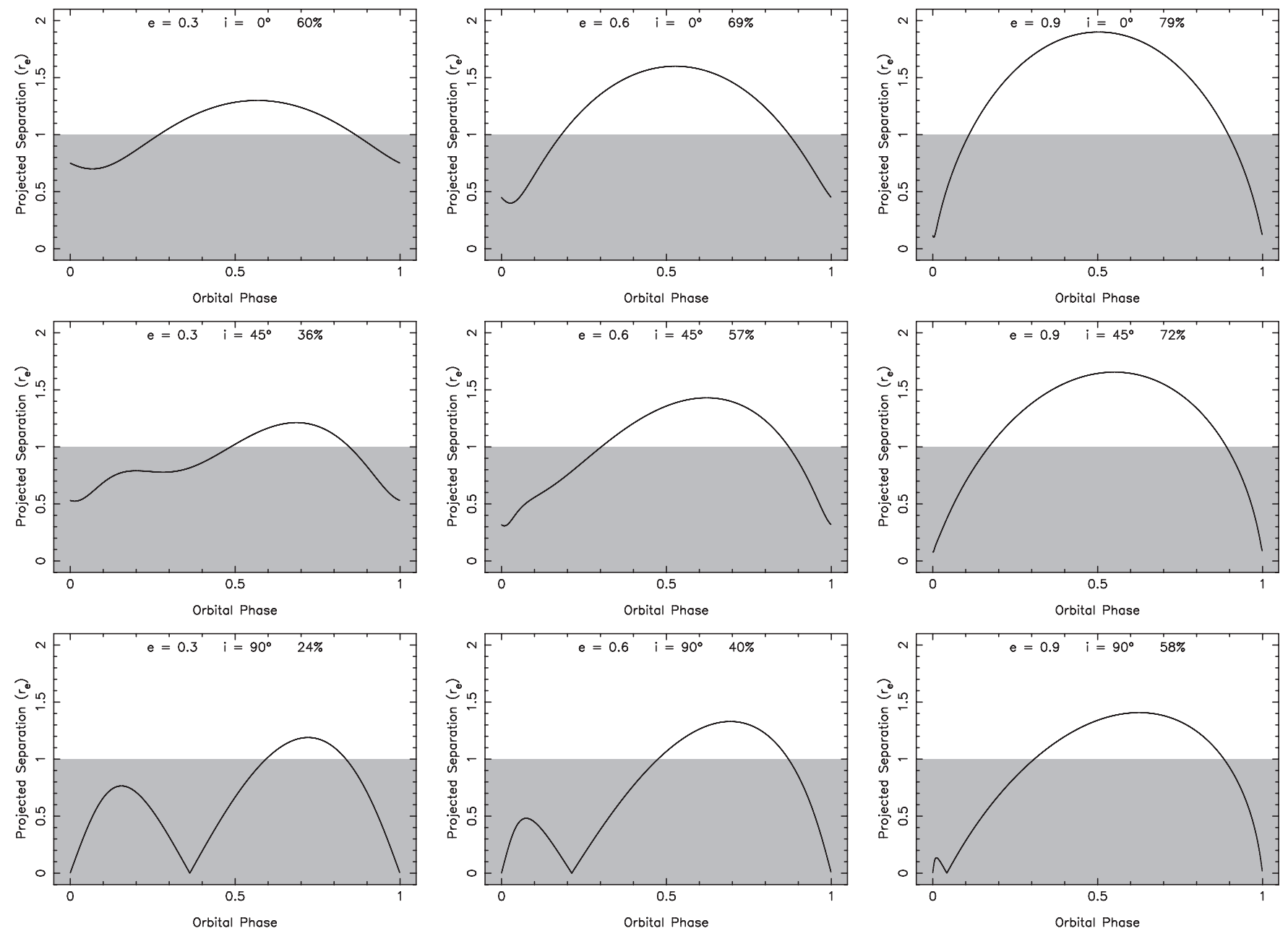

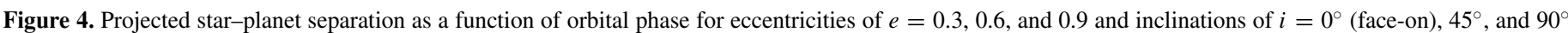

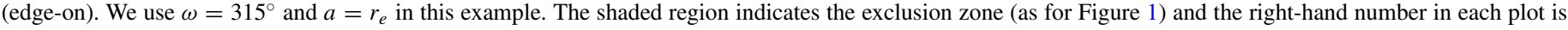
the percentage of the orbit that lies outside the exclusion zone.

it is unclear if this is an astrophysical reality or a symptom of $\mathrm{RV}$ survey incompleteness to eccentric orbits at longer periods (Cumming 2004; Shen \& Turner 2008).

\section{DISCUSSION}

There are numerous imaging surveys that have considered the detection limitations of their particular experimental designs (Chauvin et al. 2010; Bonavita et al. 2012; Brandeker et al. 2006; Delorme et al. 2012; Janson et al. 2012; Lafrenière et al. 2007a; Nielsen et al. 2008; Nielsen \& Close 2010; Vigan et al. 2012). These often treat the eccentricity distribution as a basic Gaussian function based upon the RV exoplanets. Examples of the methods explored are the commonly used probability density distribution approach described in Appendix A of Brandeker et al. (2006) and the Monte Carlo simulation code (MESS) developed by Bonavita et al. (2012). The difference with our approach here is that (1) we use the RV eccentricity distribution directly rather than infer a function from that distribution, and (2) we specifically determine the effect of eccentricity and the correlated parameters of periastron argument and inclination on detection efficiency.

The Monte Carlo simulation we perform in Section 4 uses the eccentricity distribution of RV planets at long-periods as a proxy for the expected distribution at even larger separations. However, as was noted at the conclusion of the section, it is unclear if this distribution does indeed persist in this fashion. Additionally, the samples of RV host stars and imaging survey targets tend to represent quite different stellar demographics in terms of their distances and ages. Eccentricity studies of binary stars by Duquennoy \& Mayor (1991) indicate that the distribution peaks at $e \sim 0.35$ for $P>1000$ days, whereas the mean eccentricity of the known RV planets between 1.0 and 2.0 AU is 0.27 (see Section 3). However, considering the very different formation mechanisms between stars and planets, such a comparison may be of dubious value. Constraints on the orbital eccentricity of beta Pictoris b by Chauvin et al. (2012) and on the eccentricities of the HR 8799 planets by Currie et al. (2012b) have shown that these eccentricities are relatively low $(<0.3)$. Thus the number of imaged planets is small enough and the eccentricities low enough such that little may be discerned regarding the eccentricity distribution from these planets alone. Increasing this sample by a factor of 10 would start to produce meaningful insight into the distribution of eccentricities beyond that which is currently being probed by RV surveys. As imaging experiments are able to decrease the exclusion radius and provide adequate observational cadence, the ability to detect the orbital motion of detected planets (and thus constrain 


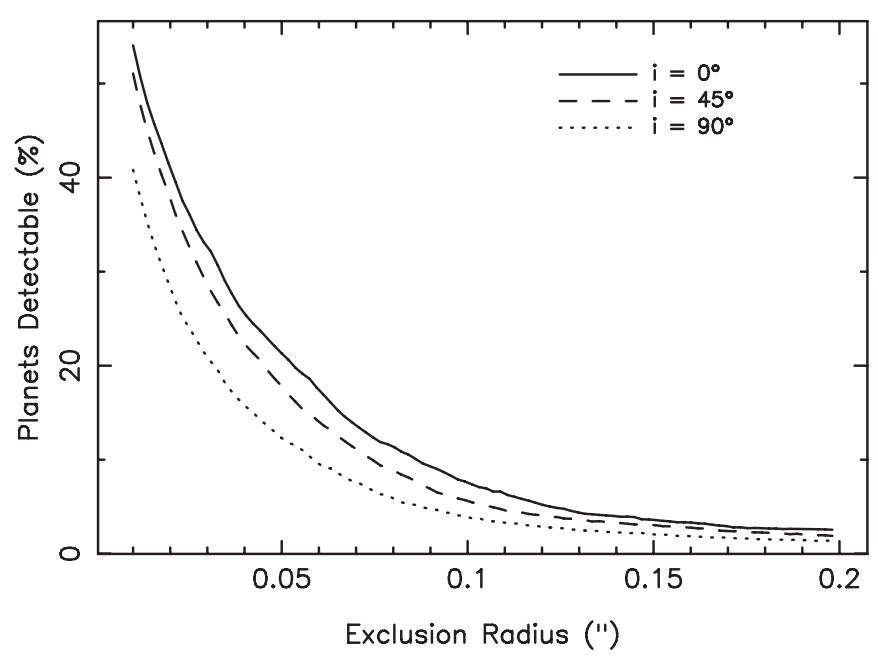

Figure 5. Results of a Monte Carlo simulation which calculates the percentage of the known exoplanets (see Section 3) which would be detected using direct imaging as a function of the exclusion radius for that particular experiment. Since we cannot assume an inclination, we have performed this simulation for inclinations of $i=0^{\circ}, 45^{\circ}$, and $90^{\circ}$.

the Keplerian properties of that orbit) will correspondingly increase.

\section{CONCLUSIONS}

The exoplanet detection method of direct imaging is a powerful technique which is rapidly developing in both technical and scientific contexts. The major frontier push is to not only to detect smaller masses, but to detect those at smaller separations from their stars. Thus the overlap between the semimajor axis parameter-space of the RV and imaging techniques is becoming larger at a rate such that it's prudent to investigate the kinds of Keplerian orbital solutions the imaging technique may expect to encounter with new discoveries. Here we have shown the impact of orbital eccentricity on exoplanet detectability through simulations, the observed distribution of Keplerian orbital parameters, and the use of a simplistic model of the exclusion zone for a particular experiment. In practice, the exclusion zone is not well-defined and is a complex function of the diffraction and speckle effects in the resulting images. The exclusion zone used here though well represents an inner region where detections are rendered virtually impossible by the flux of the star. These results show that the eccentricity can have a profound effect of the detection of eccentric exoplanets whose semimajor axis lie in the range $0.5<r_{e}<1.5$. We have also quantified the effects of inclination on these results and shown how face-on orbits can increase the detection efficiency for eccentric orbits. As the techniques for direct imaging improve and further epochs are acquired for the known imaged systems, the precise effect of eccentricity on these experiments will be become abundantly clearer and add enormously to our knowledge of the eccentricity distribution as very long orbital periods.
The author thanks Thayne Currie for several enlightening discussions on this topic, and also Natalie Hinkel for providing useful feedback on the manuscript. Thanks are also due to the anonymous referee, whose comments improved the quality of the paper. This research has made use of the Exoplanet Orbit Database and the Exoplanet Data Explorer at exoplanets.org. This research has also made use of the NASA Exoplanet Archive, which is operated by the California Institute of Technology, under contract with the National Aeronautics and Space Administration under the Exoplanet Exploration Program.

\section{REFERENCES}

Beichman, C. A., Krist, J., Trauger, J. T., et al. 2010, PASP, 122, 162 Bonavita, M., Chauvin, G., Desidera, S., et al. 2012, A\&A, 537, 67 Brandeker, A., Jayawardhana, R., \& Khavari, P. 2006, ApJ, 652, 1572 Butler, R. P., Wright, J. T., Marcy, G. W., et al. 2006, ApJ, 646, 505 Chauvin, G., Lagrange, A.-M., Beust, H., et al. 2012, A\&A, 542, 41 Chauvin, G., Lagrange, A.-M., Bonavita, M., et al. 2010, A\&A, 509, 52 Cumming, A. 2004, MNRAS, 354, 1165

Currie, T., Debes, J., Rodigas, T. J., et al. 2012a, ApJL, 760, L32

Currie, T., Fukagawa, M., Thalmann, C., Matsumura, S., \& Plavchan, P. 2012b, ApJL, 755, L34

Currie, T., Thalmann, C., Matsumura, S., et al. 2011, ApJL, 736, L33

Delorme, P., Lagrange, A. M., Chauvin, G., et al. 2012, A\&A, 539, 72

Duquennoy, A., \& Mayor, M. 1991, A\&A, 248, 485

Ford, E. B., \& Rasio, F. A. 2008, ApJ, 686, 621

Hinkley, S., Carpenter, J. M., Ireland, M. J., \& Kraus, A. L. 2011, ApJL, 730, L21

Hogg, D. W., Myers, A. D., \& Bovy, J. 2010, ApJ, 725, 2166

Janson, M., Bonavita, M., Klahr, H., \& Lafrenière, D. 2012, ApJ, 745, 4

Kalas, P., Graham, J. R., Chiang, E., et al. 2008, Sci, 322, 1345

Kane, S. R., Ciardi, D. R., Gelino, D. M., \& von Braun, K. 2012, MNRAS, 425, 757

Kane, S. R., \& Gelino, D. M. 2011, ApJ, 729, 74

Kley, W., \& Nelson, R. P. 2012, ARA\&A, 50, 211

Lafrenière, D., Doyon, R., Marois, C., et al. 2007a, ApJ, 670, 1367

Lafrenière, D., Doyon, R., Nadeau, D., et al. 2007b, ApJ, 661, 1208

Lafrenière, D., Marois, C., Doyon, R., Nadeau, D., \& Artigau, E. 2007c, ApJ, 660,770

Lagrange, A.-M., Bonnefoy, M., Chauvin, G., et al. 2010, Sci, 329, 57

Macintosh, B., Graham, J., Palmer, D., et al. 2007, CRPhy, 8, 365

Malmberg, D., \& Davies, M. B. 2009, MNRAS, 394, L26

Marois, C., Doyon, R., Racine, R., et al. 2005, JRASC, 99, 130

Marois, C., Lafrenière, D., Doyon, R., Macintosh, B., \& Nadeau, D. 2006, ApJ, 641,556

Marois, C., Macintosh, B., Barman, T., et al. 2008, Sci, 322, 1348

Marois, C., Zuckerman, B., Konopacky, Q. M., Macintosh, B., \& Barman, T. 2010, Natur, 468, 1080

Moorhead, A. V., Ford, E. B., Morehead, R. C., et al. 2011, ApJS, 197, 1

Namouni, F. 2005, AJ, 130, 280

Nielsen, E. L., \& Close, L. M. 2010, ApJ, 717, 878

Nielsen, E. L., Close, L. M., Biller, B. A., Masciadri, E., \& Lenzen, R. 2008, ApJ, 674, 466

Oppenheimer, B. R., \& Hinkley, S. 2009, ARA\&A, 47, 253

Quanz, S. P., Birkmann, S. M., Apai, D., Wolf, S., \& Henning, T. 2012, A\&A, 538A, 92

Racine, R., Walker, G. A. H., Nadeau, D., Doyon, R., \& Marois, C. 1999, PASP, 111,587

Ribas, I., \& Miralda-Escudé, J. 2007, A\&A, 464, 779

Shen, Y., \& Turner, E. L. 2008, ApJ, 685, 553

Vigan, A., Moutou, C., Langlois, M., et al. 2010, MNRAS, 407, 71

Vigan, A., Patience, J., Marois, C., et al. 2012, A\&A, 544, 9

Wright, J. T., Fakhouri, O., Marcy, G. W., et al. 2011, PASP, 123, 412 